\title{
Erfolgsdimensionen von Social Software: Eine fallstudienbasierte Untersuchung
}

\author{
Melanie Steinhüser' ${ }^{1}$ Philip Räth² \\ ${ }^{1}$ Lehrstubl für BWL/Organisation und Wirtschaftsinformatik, \\ Universität Osnabrück \\ 2Supply Chain Management Institute, \\ European Business School (ebs), Oestrich-Winkel
}

\section{Einleitung}

In den vergangenen zehn Jahren hat sich Social Software von einer neuen Technologie hin zu einem Massenphänomen entwickelt. Trotz einiger Kritik der klassischen Medien erfreuen sich die Anwendungen einer stetig wachsenden Popularität. Der Erfolg im privaten Umfeld hat gleichsam die Aufmerksamkeit von Organisationen auf sich gezogen. So werden Tools wie Wikis und Weblogs in unterschiedlichen Bereichen der Unternehmenskommunikation genutzt, um Mitarbeiter zu vernetzen und die Kooperation zu verbessern. Einer weltweit durchgeführten Studie von Forrester Research zufolge werden Organisationen in den nächsten Jahren umfangreiche Investitionen in diesem Bereich tätigen (Young 2007). Unternehmen berichten aber von Problemen bezüglich der Implementierung und Akzeptanz (Bughin et al. 2008). Aufgrund limitierter Budgets müssen die IT Abteilungen und Entscheidungsträger ihre Investitionen selbstverständlich begründen und daher Transparenz hinsichtlich des erwarteten Nutzens neuer Informationssysteme schaffen. Demzufolge ist es wichtig, den Erfolg von Social Software in Unternehmen zu bemessen und Faktoren zu identifizieren, die diesen beeinflussen.

Bisher haben sich nur wenige empirische Studien mit dem Einsatz und Erfolg von Social Software in Unternehmen befasst, die entweder sehr spezifische Facetten beleuchten oder einer theoretischen Grundlage entbehren. Ein fundierter Beitrag zur Theoriebildung fehlt bisher (siehe auch Majchrzak 2009). Demzufolge adressieren wir folgende Forschungsfragen: Warum setzen Organisationen Social Software ein? Und welches sind die erfolgsrelevanten Faktoren einer Social Software Implementierung?

Wir nehmen an, dass die existierenden Modelle zur Erfolgsmessung von Informationssystemen, die typischerweise herkömmliche, eher technisch orientierte Software wie ERP-Systeme betrachten, sich aufgrund der besonderen Eigenschaften von Social Software (siehe Abschnitt 2.1) nicht ohne Modifikationen anwenden 
lassen. Vor dem Hintergrund, dass Social Software, ein relativ junger Forschungsgegenstand ist, ist eine systematische Untersuchung explorativen Charakters nötig. Hierzu wurden Fallstudien in Unternehmen durchgeführt, die unterschiedliche Social Software Anwendungen in der internen Unternehmenskommunikation nutzen. Diese liefern empirische Ergebnisse zu den o.a. Fragen, die Schlüsse bzgl. der Nutzung und den erfolgsrelevanten Gestaltungsparametern zulassen. Wir schlagen Maßnahmen vor, ein geeignetes Instrument zur Erfolgsmessung von Social Software zu entwickeln, wobei das IS-Impact Measurement Model (Gable et al. 2008) als theoretischer Rahmen dient.

\section{Grundlagen}

\subsection{Social Software}

Auf einer sehr allgemeinen Ebene werden unter Social Software webbasierte Anwendungen zusammengefasst, die die menschliche Interaktion (Kommunikation, Koordination, Kooperation) sowie die Vernetzung der User unterstützen (Cook 2008, S. 39; Koch und Richter 2007, S. 14). Prinzipiell ist Social Software kein völlig neues Phänomen; Social Computing, Groupware und Computer Supported Cooperative Work (CSCW) sind einander ähnelnde Konzepte, die in der Wissenschaft seit den 80er Jahren untersucht werden. Die Nutzer interagieren digital, erstellen Inhalte und strukturieren (gliedern, bewerten, verschlagworten) Informationen gemeinsam. Grundsätzlich ist Social Software einfach zu benutzen, intuitiv zu bedienen und erfordert daher im Vergleich zu anderen Unternehmensanwendungen verhältnismäßig wenig Schulungen und IT Support (Lai und Turban 2008, S. 388-389; Parameswaran und Whinston 2007, S. 337-338). Die Interaktion ist durch eine n:m Beziehung gekennzeichnet (Cook 2008, S. 9). Dabei gibt die Software an sich keine Struktur vor, sondern folgt dem Prinzip der Selbstorganisation, weswegen sie auch als Bottom-up Technologie bezeichnet wird (McAfee 2006, S. 25; Parameswaran und Whinston 2007, S. 337). Das „Neue“ oder „Andere“ an Social Software unterstreichen Bouman et al. (2007, S. 2) mit folgender Aussage: „Sociality, not functionality, is the key concept".

In unserer Untersuchung haben wir uns auf Wikis und Blogs konzentriert, deren Eigenschaften und Funktionalitäten wir im Folgenden kurz erläutern: Leuf und Cunningham (2008, S. 14) definieren ein Wiki als unbegrenzt erweiterbare Sammlung miteinander verlinkter Webseiten zum Speichern und Bearbeiten von Informationen. Beiträge können auf einfache Weise durch jeden Nutzer erstellt, editiert, erweitert und kommentiert werden. Da Wikis in der Regel über Such- und Indizierungsfunktionalitäten verfügen, unterstützen sie auch beim Auffinden und der weiteren Nutzung von Informationen (Hester und Scott 2008, S. 2). Die kollaborative Arbeit sowie Feedbackfunktionalitäten und die Versionskontrolle können zu einer Qualitätssicherung der Inhalte führen, die auf Teamgeist und 
Kreativität basiert anstatt auf Kontrolle (Wagner und Majchrzak 2006, S. 20). Unternehmen setzen Wikis in zunehmendem Maße ein, um die Zusammenarbeit der Mitarbeiter und das Wissensmanagement zu unterstützen (Majchrzak 2009, S. 18). Auch Weblogs (Blogs) finden immer häufiger Einzug in die Berufswelt und werden von Unternehmen zur internen und externen Kommunikation genutzt (Efimova und Grudin 2007). Blogs sind Webseiten, auf denen ein Autor bzw. eine kleine Gruppe von Autoren Texte oder andere Dokumente in regelmäßigen oder unregelmäßigen Abständen veröffentlicht. Kennzeichnend für Blogeinträge, privat wie beruflich, ist dabei der persönliche Stil sowie die Perspektive des/der Autoren (Wattal et al. 2009, S. 2). Besucher können diese Beiträge kommentieren, was zu lebhaften Diskussionen im Blog und auch außerhalb dieses führen kann. Die Inhalte sind chronologisch sortiert, eine Einordnung in Themenbereiche erfolgt meist zusätzlich. Blogs sind in sich und mit anderen Blogs verlinkt, wobei jeder Beitrag stets unter einer spezifischen URL (Permalink) abrufbar ist. RSS und andere Feed-Formate wie Atom ermöglichen Lesern die automatische Syndizierung verschiedener Blogs. Mitarbeiter nutzen Blogs als Medium, ihre Expertise zu teilen, Meinungen zu äußern und Diskussionen anzuregen, was die Möglichkeit bietet, die virtuelle Zusammenarbeit innerhalb von Organisationen und darüber hinaus effizienter zu gestalten (Trimi und Galanxhi-Janaqi 2008, S. 125).

\subsection{Erfolgsmessung von Informationssystemen und von Social Software}

Während die Messung des Erfolges von Informationssystemen als wichtiges Thema betrachtet und in Organisationen viel diskutiert wird (z. B. Irani und Love 2000), gibt es wenig Konsens darüber, wie dies zuverlässig geschehen kann. In der Wissenschaft dominiert das DeLone \& McLean IS Success Model (DeLone und McLean 1992; DeLone und McLean 2003), welches in seiner überarbeiteten Form die folgenden sechs Dimensionen umfasst: Systemqualität, Informationsqualität, Servicequalität, Systemnutzung, Nutzerzufriedenheit und Nettonutzen. Das DeLone \& McLean IS Success Model ist eines der am häufigsten zitierten und angewandten Modelle der Erfolgsmessung in der Wirtschaftsinformatik, da es einen übersichtlichen Rahmen und validierte Maße bietet. Im Rahmen einer umfangreichen Literaturanalyse zeigen Urbach et al. (2008) die Dominanz des Modells und geben einen Überblick über bisherige Einsatzszenarien. Gleichwohl existiert trotz dieser Popularität eine Reihe von Kritikpunkten an dem Modell. Diese beziehen sich auf die fehlende theoretische Fundierung, einen zu starken Fokus auf monetäre Kennzahlen, sich überschneidende Dimensionen, unscharfe Maße sowie den Versuch, Prozess- und Kausalmodell in einem zu vereinen (Ballantine et al. 1996; Seddon 1997).

Gable et al. (2008) haben daraufhin das IS-Impact Measurement Model (Abbildung 1) eingeführt, welches auf der Arbeit von DeLone und McLean basiert, aber versucht, einigen der Kritikpunkte entgegenzuwirken (siehe auch Sedera und Gable 2004). So schließen die Autoren aus eigenen Studien (sowie in Überein- 
stimmung mit anderen Wissenschaftlern, wie Rai et al. 2002 oder Sedera und Tan 2005), dass die von DeLone und McLean verwendeten Dimensionen Use und User Satisfaction lediglich einen Vorgänger sowie eine Konsequenz des IS-Impact darstellen, weshalb diese Konstrukte nicht als erfolgsrelevante Dimensionen in ihr Modell aufgenommen wurden.

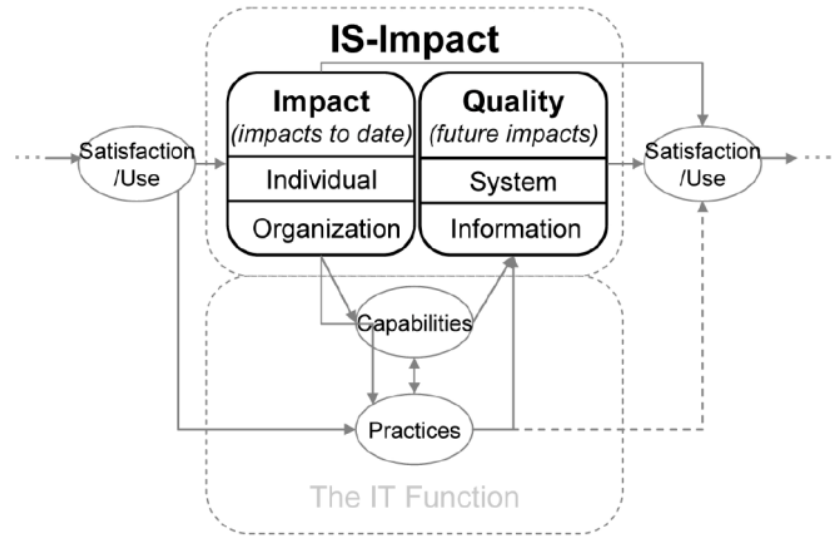

Abbildung 1: IS-Impact Measurement Model (Quelle: Gable et al. 2008, S. 395)

Demgemäß kann die Messung des Erfolgs eines Informationssystems, anhand von vier Dimensionen erfolgen: Impact umfasst dabei aktuelle individuelle (Individual Impact) sowie organisatorische (Organizational Impact) Einflüsse, während Quality die künftige System- (System Quality) und Informationsqualität (Information Quality) beinhaltet. Individual Impact beschreibt, inwiefern das Informationssystem die Effektivität von Nutzern beeinflusst. Mit Organizational Impact wird erklärt, inwieweit das Informationssystem für eine Verbesserung der Ressourcen und der organisatorischen Ergebnisse sorgt. Mit Information Quality wird die Qualität der Systemausgabe aufgezeigt, während System Quality eine Messung der technischen und designbasierten Performanz des Systems darstellt. Unter Berücksichtigung sich möglicherweise überschneidender Maße, evaluierten die Autoren bereits existierende Maße, haben Redundanzen aufgelöst und neue Faktoren identifiziert. Im Zuge der Validierung des Modells reduzierten sie die Anzahl relevanter Maße von zunächst 37 infrage kommenden auf 27 Erfolgsfaktoren.

Das IS-Impact Mesurement Model tritt damit einigen Unklarheiten und Schwachstellen des DeLone \& McLean IS Success Model entgegen; es bietet valide Maßzahlen und einen nachvollziehbaren Ansatz, der Rückschlüsse aus unterschiedlichen Perspektiven zulässt. Die Autoren erklären, dass das Modell in modifizierter Form Vergleiche über unterschiedliche Systeme und Kontexte zulässt. In vorigen Abschnitt haben wir die Besonderheiten hervorgehoben, die Social Software von herkömmlichen Systemen unterscheiden. Diese lassen den Schluss zu, dass das IS Impact Measurement Model sich ohne Modifikationen nicht auf Social Software übertragen lässt. 
In Abschnitt 2.1 haben wir die Besonderheiten hervorgehoben, die Social Software von Systemen wie ERP unterscheiden. Diese lassen den Schluss zu, dass bekannte Modelle, die den Erfolg von Informationssystemen beschreiben, sich ohne Modifikationen nicht auf Social Software übertragen lassen. Wie bei anderen Informationssystemen, so kann der Erfolg von Social Software ebenfalls aus unterschiedlichen Perspektiven betrachtet werden. Bisher gibt es wenig wissenschaftliche Veröffentlichungen, die sich mit diesem Thema auseinandersetzen, und keine, die versucht, einen Rahmen zu spannen. Um einen Überblick über den Stand der Forschung zu erhalten, haben wir eine umfangreiche Literaturrecherche durchgeführt. Dabei haben wir einige Arbeiten identifiziert, die erfolgsrelevante Faktoren betrachten (Du und Wagner 2006; Hester und Scott 2008; Hsu und Lin 2008; Ip und Wagner 2008; Räth et al. 2009; Reisberger 2008; Trimi und Galanxhi-Janaqi 2008), aber ausschließlich spezielle Aspekte des Erfolgs fokussieren oder einer theoretischen bzw. empirischen Fundierung der Erkenntnisse entbehren. Die Entwicklung eines umfassenden Instrumentes zur Erfolgsmessung von Social Software basierend auf fundierten Erkenntnissen steht somit noch aus.

\section{Datenerhebung und Analyse}

Um ein geeignetes Messinstrument für den Erfolg von Social Software zu entwickeln, ziehen wir das oben beschriebene IS-Impact Measurement Model als Rahmen und Ausgangspunkt unserer empirischen Forschung heran. Aufgrund der schwachen empirischen Grundlage im Bereich Social Software, wählen wir eine explorative Forschungsmethodik. Diese ermöglicht, Dimensionen und Faktoren zu berücksichtigen, die in dem Modell bisher nicht erfasst wurden. Besonders die Fallstudienforschung eignet sich, Bereiche zu untersuchen, zu denen kein umfangreiches empirisches Material vorliegt. Bzgl. des Forschungsprozesses haben wir uns dabei an dem von Eisenhardt (1989) vorgeschlagenen Vorgehen zur Erhebung und Auswertung von Fallstudien orientiert. Drei Unternehmen unterschiedlicher Branchen wurden in die Studie einbezogen (Tabelle 1).

Tabelle 1: Charakteristika der an den Fallstudien beteiligten Unternehmen

\begin{tabular}{|l|c|c|c|}
\hline & Unternehmen 1 & Unternehmen 2 & Unternehmen 3 \\
\hline Branche & Energie- und Automation & Flughafendienstleistungen & Medizintechnik \\
\hline Umsatz in $T €$ & 24.328 & 2.106 & 6.470 \\
\hline Mitarbeiter & 120.000 & 23.000 & 32.500 \\
\hline Social Software & Weblog und Wiki & Wiki & Weblog und Wiki \\
\hline
\end{tabular}

Die Daten wurden zwischen April und September 2009, wie von Yin (2003) vorgeschlagen, anhand unterschiedlicher Techniken erhoben und in die Analyse einbezogen. Dies waren semi-strukturierte Interviews, Dokumente, interne Präsentationen, Internetseiten sowie Nutzerstatistiken. Die Interviewpartner waren Mitarbeiter, die sowohl aktive als auch passive Nutzer der Systeme sind. Das heißt, dass sie 
sowohl Informationen in das System eingeben, als auch Output aus den Systemen erhalten. In den explorativen Interviews haben wir nach erfolgsrelevanten Faktoren der Social Software gefragt. Dies umfasste die Thematisierung der Definition von Erfolg sowie der Faktoren, die diesen Erfolg beeinflussen und anhand derer sich ein Erfolg messen lassen kann.

Die geführten Interviews haben wir transkribiert und codiert. Zusammen mit anderen Dokumenten und Informationen wurde jede Fallstudie zusammengefasst, analysiert und Schlussfolgerungen daraus getroffen. Die transkribierten Interviews haben wir in kurze Sätze isoliert, die wir Impact Citation nennen. 266 Zitate wurden aus den Antworten der Interviewten isoliert. Diese Impact Citations wurden in relevante IS-Impact Maße gruppiert. (Wir sind dem Rat der Autoren des IS-Impact Measurement Model gefolgt, die empfehlen, alle $37 \mathrm{Maße}$ zu berücksichtigen, wenn in einem neuen Kontext geforscht wird.) Jede Aussage musste dabei explizit eine Information über den Einfluss der Social Software beinhalten. Dieser Prozess des Isolierens und Gruppierens wurde für jede Fallstudie von einem Wissenschaftler durchgeführt, der auch in die Datenerhebung involviert war. Um die Qualität der Daten nicht durch Quantität ersetzten zu müssen, wurden die verschiedenen Nennungen nicht ausgezählt, sondern nach qualitativen Maßstäben gewichtet. Dabei haben die beteiligten Forscher die Aussagen bewertet, indem sie nicht nur die Häufigkeit der Nennung berücksichtigten, sondern als ein weiteres Kriterium wertende Aussagen der Interviewten (z. B. ,...als sehr wichtig wurde erachtet...“ oder ,... war entscheidend...") sowie weitere zur Verfügung stehende Dokumente berücksichtigt. Durch Diskussionen innerhalb der Forschungsgruppe wurden die Aussagen schließlich mit wichtig $(+)$, sehr wichtig $(++)$ oder unwichtig $(\mathrm{O})$ bewertet. Anschließend wurde eine Analyse durchgeführt, in der wir sowohl nach fallinternen wie auch nach fallübergreifenden Mustern suchten. Ein Überblick über die Ergebnisse findet sich im nächsten Abschnitt.

\section{Ergebnis}

Die Einordnung der in den Fallstudien identifizierten erfolgsrelavanten Faktoren in die Dimensionen des IS-Impact Measurement Model ist in Tabelle $2 \mathrm{zu}$ sehen. Bezüglich der Dimension Individual Impact haben wir festgestellt, dass alle vom Modell vorgeschlagenen Maße auch für die Erfolgsmessung von Social Software relevant sind. Besonders die Awareness ist an dieser Stelle hervorzuheben, da dieser Punkt in allen drei Fallstudien als mindestens wichtiger Erfolgsfaktor identifiziert wurde. Das Bewusstsein um die Aktivitäten (und den Status) der Personen, die miteinander arbeiten wird durch Funktionalitäten von Social Software, wie die Historie in Wikis oder Push Funktionalitäten bei Blogs, unterstützt. 
Tabelle 2: Identifizierte Erfolgsfaktoren und -dimensionen

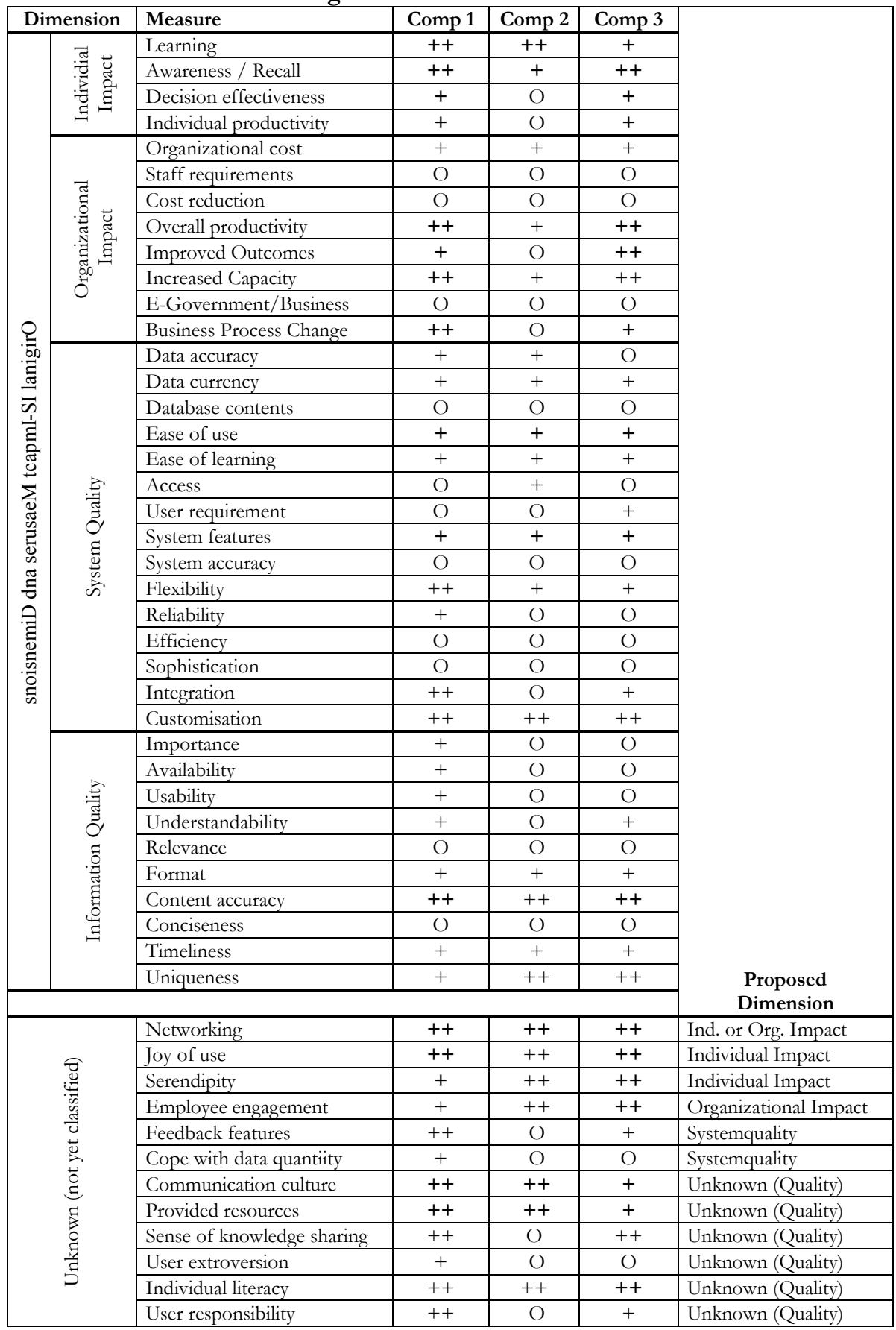


Die Ergebnisse lassen hinsichtlich der Dimension Organizational Impact den Schluss $\mathrm{zu}$, dass einige der in dem Modell befindlichen Maße bei der Nutzung von Social Software nicht bedeutsam sind. Während bspw. die Reduktion von Verwaltungskosten nicht als relevant eingestuft wurde, so sind Verbesserungen hinsichtlich der Geschäftsprozesse sowie der Produktivität und der Arbeitsergebnisses durchaus Ziele, die mit dem Einsatz von Social Software verfolgt werden. In die Dimension System Quality fließen viele Maße ein, die nachvollziehbar auch hinsichtlich Social Software eine wichtige, aber, aufgrund der technischen Schlichtheit der Systeme, selten eine sehr wichtige Rolle spielen. Hervorzuheben sind an dieser Stelle die besondere Relevanz der individuellen Anpassbarkeit, sowie die immer noch bedeutsame, aber vergleichsweise wenig wichtige Rolle des Faktors Nutzerfreundlichkeit. Aussagen der Interviewten und der Fakt, dass die Einfachheit der Nutzung quasi schon per Definition zu Social Software gehört, lassen schließen, dass dies ein Punkt ist, der von der Software erwartet wird, und somit bei Vorhandensein keinen sehr großen Einfluss mehr auf den Erfolg ausübt. Hinsichtlich Information Quality ist besonders die Validität der Inhalte (Content Accuracy) zu betonen. Aufgrund der Offenheit und der unkontrollierten Veränderbarkeit haben die Befragten Vertrauen in die Qualität der Beiträge als sehr wichtig erachtet. Andere Maße wie Relevanz und Prägnanz der Inhalte verlieren in Bezug auf Social Software an Bedeutung.

12 Konstrukte wurden identifiziert, die sich in den Maßen des IS-Impact Measurement Model nicht widerspiegeln. Einige lassen sich in die Dimensionen des Modells einordnen. So wurden bspw. Spaß und Serendipity als (sehr) wichtige erfolgsrelevante Auswirkungen identifiziert, die der Dimension Individual Impact zugeordnet werden können. Ähnlich verhält es sich mit dem Faktor Mitarbeiterbindung, der sich zur Dimension Organizational Impact gruppieren kann. Durchweg als sehr wichtig wurde die Vernetzung der Mitarbeiter bewertet. Inwieweit dies den organisationalen oder den individuellen Nutzen betrifft, ist je nach Betrachtungsweise unterschiedlich und muss daher gegebenenfalls in beiden Dimensionen Individual Impact und Organizational Impact Berücksichtigung finden. Interessant ist in diesem Zusammenhang, dass die Vernetzung als explizites Ziel identifiziert wurde, was künftige Überlegungen hinsichtlich der Zuordnung von Punkten wie Serendipity, Kommunikationsgeschwindigkeit und Awareness erfordert.

Sechs Faktoren wurden aus den Daten gewonnen, die als (sehr) erfolgsrelevant bewertet wurden, und sich nicht in die vier Dimensionen des IS-Impact Measurement Model eingliedern lassen. Sie betreffen zwar durchweg die Qualität des Informationssystems, fallen aber weder unter die technisch orientierte Dimension System Quality noch unter Information Quality. Dies sind: Unternehmens- und Kommunikationskultur, verfügbare Ressourcen, Bereitschaft, Wissen zu teilen, Extravertiertheit der Nutzer, Literacy sowie Verantwortung. Dabei umfasst die Unternehmens- und Kommunikationskultur das Arbeitsklima innerhalb einer Unternehmung und bezieht sich darauf, inwieweit die Meinung der Mitarbeiter zählt und offene Diskussionen und Kritik erwünscht sind. Darüber hinaus ist es von ent- 
scheidender Bedeutung, dass den Mitarbeitern Ressourcen (vor allem Zeit) für die Nutzung der Systeme zur Verfügung stehen. Sicherlich von der Unternehmenskultur abhängig, aber eher auf einer individuellen Ebene wiederzufinden, ist die Bereitschaft der Nutzer, ihr Wissen anderen offen zur Verfügung zu stellen. Eine Arbeitspraxis, die auf dem Gedanken beruht, dass Wissen = Macht bedeutet, ist dabei hinderlich. Eine Form der individuellen Extravertiertheit, aufgrund derer die Nutzer nach außen gerichtete Kommunikation als anregend empfinden, wurde ebenfalls als erfolgsrelevant bezeichnet. Unter Literacy fällt die Fähigkeit der aktiv beitragenden Nutzer, Beiträge zu verfassen, die weder in zu geschäftsmäßigem Stil noch Arbeitsirrelevantes oder -unpassendes behandeln. Schließlich wurde in zwei Fallstudien die Übernahme von Verantwortung durch die User als (sehr) wichtig für den Erfolg eingestuft. Wikis und Blogs setzen auf eine freiwillige Nutzung, Kontrollinstanzen sind unüblich und auch in den von uns analysierten Unternehmen nicht vorhanden. Die Verantwortung über die Qualität der Inhalte geht also auf die Nutzer über, was einen entsprechenden Umgang erforderlich macht.

\section{Diskussion}

Die Ergebnisse unserer Studie liefern wichtige Hinweise auf Erfolgsfaktoren, die bei der Implementierung und Nutzung von Social Software eine entscheidende Rolle spielen. Im Zuge der Analyse wurden einerseits neue Faktoren identifiziert, die für den Erfolg von Social Software relevant sind, aber durch das IS-Impact Measurement Model nicht erklärt werden. Auf der anderen Seite erscheinen einige Maße des Modells in einem Social Software Kontext nicht relevant und können bei der Erfolgsmessung außen vor bleiben. Von den neu identifizierten Faktoren lassen sich einige in die bestehenden Dimensionen einordnen, es wird aber deutlich, dass das Modell ohne weitere Modifikationen nicht zur Erfolgsmessung von Social Software herangezogen werden kann. Vielmehr muss eine neue Social Software spezifische Dimension hinzugefügt werden, da gerade hier Interaktionen wie Kommunikation und Kollaboration den Erfolg positiv beeinflussen können (Burton-Jones und Gallivan 2007). Das IS-Impact Measurement Model hat zwar den Anspruch, auf unterschiedliche Systeme anwendbar zu sein, wurde aber (wie auch das DeLone \& McLean IS Success Model) unter Annahmen klassischer Informationssysteme (wie ERP) entwickelt und validiert. Es spiegelt somit eine eher technisch orientierte Perspektive wider und vernachlässigt soziale Aspekte, die sich auf die Nutzung und den Erfolg auswirken. Wie wir gezeigt haben, ist eine soziotechnische Betrachtung in einem Social Software Kontext aber nicht zu vernachlässigen. Das heißt, dass Dimensionen etablierter Modell wie System Quality und Information Quality zwar weiterhin wichtig sind, aber nicht ausreichen, um den Erfolg von Social Software umfassend und zuverlässig zu bemessen.

Es gilt also, eine neue Dimension aufzunehmen, wenn es um die Erfolgsmessung von Social Software geht. Auf dem Weg zu einer theoriegeleiteten Entwick- 
lung einer solchen neuen Dimension lassen sich ggf. andere Theorien, die bisher bei Erfolgsmodellen nicht berücksichtigt wurden, heranziehen. Forschung in Bereichen wie Knowledge Sharing haben gezeigt dass Theorien wie die Social Capital Theorie und die Social Exchange Theorie Erklärungsmuster für soziale Phänomene bieten (McLure-Wasko Faraj 2005; Majchrzak 2009). Andere Ansätze liefern Strukturationstheorien, die aufzeigen, wie Technologien und Organisationen sich gegenseitig beeinflussen und dadurch zu verschiedenen Ergebnissen führen (DeSantics und Poole 1994). Jüngste Forschungen im Bereich Multilevel Analysen (Burton-Jones und Gallivan 2007) eröffnen zudem Möglichkeiten, die Komplexität der Nutzung und des Erfolgs von Social Software abzubilden.

Obwohl die Fallstudien nicht repräsentativ sind, so haben sie doch aufgrund ihres explorativen Charakters einige interessante Ergebnisse hervorgebracht. Das IS-Impact Measurement Model wird in Teilen bestätigt, in wichtigen Bereichen muss es angepasst werden, um korrekte Aussagen bezüglich des Erfolgs von Social Software treffen zu können. Ergebnisse unserer Studie gelten zunächst für Weblogs und Wikis, lassen sich aber gegebenenfalls aufgrund der erläuterten Gemeinsamkeiten auch auf andere Anwendungen übertragen. Um diesen Einschränkungen zu begegnen, planen wir in nächster Zeit, weitere Unternehmen zu untersuchen, die Social Software unterschiedlicher Art einsetzen.

\section{Literatur}

Ballantine J, Bonner M, Levy M, Martin A, Munro I, Powell, P (1996) The 3-D model of information systems success: The search for the dependent variable continues. Information Resources Management Journal 9(4): 5-14.

Bouman W, Bruin B, Hoogenboom T, Huizing A, Jansen R, Schoondorp M (2007) The realm of sociality: Notes on the design of social software. In: Proceedings of the International Conference on Information Systems, Montréal.

Bughin J, Manyika J, Miller A (2008) Building the web 2.0 enterprise. McKinsey Global Survey Results. In: McKinsey Quarterly.

Burton-Jones A, Gallivan MJ (2007) Toward a Deeper Understanding of System Usage in Organizations: A Multilevel Perspective. MIS Quarterly. 31 (4): 657679

Cook N (2008) Enterprise 2.0. How social software will change the future of work. Gower, Aldershot.

DeLone WH, McLean ER (1992) Information systems success: The quest for the dependent variable. Information Systems Research 3(1): 60-95. 
DeLone WH, McLean ER (2003) The DeLone and McLean model of information systems success: A ten-year update. Journal of Management Information Systems 19(4): 9-30.

DeSantics G, Poole MS (1994) Capturing the Complexity in Advanced Technology Use: Adaptive Structuration Theory. Organization Science 5(2): 421-147.

Du HS, Wagner C (2006) Weblog success: Exploring the role of technology. In: International Journal of Human-Computer Studies 64(9): 789-798.

Efimova L, Grudin J (2007) Crossing boundaries: A case study of employee blogging. In: Proceedings of the 40th Annual Hawaii International Conference on System Sciences, Hawaii.

Eisenhardt KM (1989) Building theories from case study research. Academy of Management Review 14(4): 532-550.

Gable GG, Sedera D, Chan T (2008) Re-conceptualizing information system success: The IS-impact measurement model. Journal of the AIS 9(7): 377-408.

Hester AJ, Scott JE (2008) A conceptual model of wiki technology diffusion. In: Proceedings of the 41st Annual Hawaii International Conference on System Sciences, Hawaii.

Hsu C, Lin J (2008) Acceptance of blog usage. The roles of technology acceptance, social influence and knowledge sharing motivation. Information \& Management 45(1): 65-74.

Ip RKF, Wagner C (2008) Weblogging: A study of social computing and its impact on organizations. Decision Support Systems 45(2): 242-250.

Irani Z, Love PED (2000) The propagation of technology management taxonomies for evaluating investments in information systems. Journal of Management Information Systems 17(3): 161-177.

Koch M, Richter A (2007) Enterprise 2.0. Planung, Einführung und erfolgreicher Einsatz von Social Software in Unternehmen. Oldenbourg, München.

Lai LSL, Turban E (2008) Groups formation and operations in the web 2.0 environment and social networks. In: Group Decision and Negotiation 17(5): 387-402.

Leuf B, Cunningham W (2008) The wiki way. Quick collaboration on the web. Addison-Wesley, Boston.

Majchrzak A (2009) Comment: Where is the theory in wikis? MIS Quarterly 33(1): 18-20.

McAfee AP (2006) Enterprise 2.0: The dawn of emergent collaboration. MIT Sloan Management Review 47(3): 21-28. 
McLure-Wasko M Faraj S (2005) Why Should I Share? Examining Social Capital and Knowledge Contribution in Electronic Networks of Practice. MIS Quarterly (29: 1).

Parameswaran M, Whinston AB (2007) Research issues in social computing. Journal of the AIS 8(6): 336-350.

Rai A, Lang SS, Welker RB (2002): Assessing the validity of IS success models: An empirical test and theoretical analysis. Information Systems Research 13(1): 50-69.

Räth P, Smolnik S, Urbach N, Zimmer C (2009) Towards assessing the success of social software in corporate environments. In: Proceedings of 15 th Americas Conference on Information Systems, San Francisco.

Reisberger T, Smolnik S (2008) Modell zur Erfolgsmessung von Social-SoftwareSystemen. In: Tagungsband der Multikonferenz Wirtschaftsinformatik, München.

Seddon PB (1997) A respecification and extension of the DeLone and McLean model of IS success. Information Systems Research 8(3): 240 -253.

Sedera D, Gable GG (2004) A factor and structural equation analysis of the enterprise systems success measurement model. In: Proceedings of the International Conference on Information Systems, Washington, DC.

Sedera D, Tan F (2005) User satisfaction: a dimension of enterprise system success. In: Proceedings of the Ninth Pacific Asia Conference on Information Systems.

Trimi S, Galanxhi-Janaqi H (2008) Organisation and employee congruence: a framework for assessing the success of organisational blogs. International Journal of Information Technology and Management 7(2): 120-133.

Urbach N, Smolnik S, Riempp G (2008) A methodological examination of empirical research on information systems success: 2003 to 2007. In: Proceedings of 14th Americas Conference on Information Systems, Toronto.

Wagner C, Majchrzak A (2006) Enabling customer-centricity using wikis and the wiki way. Journal of Management Information Systems 23(3): 17-43.

Wattal S, Racherla P, Mandviwalla M (2009) Employee adoption of corporate blogs: A quantitative analysis. In: Proceedings of the 42nd Hawaii International Conference on System Sciences, Hawaii.

Yin RK (2003) Case study research. Design and methods. Sage, Thousand Oaks.

Young GO (2007) Global enterprise web 2.0 market forecast: 2007 to 2013. Forrester Research. 